\title{
Design issues of the piezo motor for the spacecraft reflector control system
}

\author{
Anton Azin ${ }^{1, *}$, Sergey Rikkonen ${ }^{1}$, Sergey Ponomarev ${ }^{1}$, and Stanislav Kuznetsov ${ }^{2}$ \\ ${ }^{1}$ National Research Tomsk State University, Research Institute of Applied Mathematics and \\ Mechanics, 634050 Tomsk, Russia \\ ${ }^{2}$ Academician M.F. Reshetnev Information Satellite Systems,662972 Zheleznogorsk, Krasnoyarsk \\ region, Russia
}

\begin{abstract}
Creation of large-size reflectors for spacecrafts is a topical issue for the space industry. The accuracy of the reflecting surface form and the structure weight are the main criteria for the reflector design. The accuracy of the reflecting surface form during a long-term operation is provided by adjustment when using piezoelectric motors in the reflector design. These motors have small weight-size parameters and can reach great torque values. The piezo motor is a distributed mechanical-acoustic oscillation system. Mechanical-acoustic oscillations are generated in the piezo motor by a PZT-stack and transmitted to an oscillator element, and then from the oscillator element to a load action element. At high frequencies, when dimensions of the oscillator are proportionate to the wavelength, the energy is transmitted by means of acoustic waves. In this case, mechanical waves practically are not involved in the energy transmission process. This thesis shows a method for selecting the material of a mechanical-acoustic oscillation system according to the efficiency of the acoustic energy transmission via a piezoelectric layered structure.
\end{abstract}

\section{Introduction}

Reduction of weight-size parameters of the spacecraft (SC) systems is the key issue in the space industry. One of the solutions is substitution of electromechanical drives of different SC devices for a variety of piezo motors, while weight-size parameters of the corresponding devices are reduced by several times. PZT-stack based piezo motors are the most reliable and powerful ones $[1,2]$.

The piezo motor is a distributed mechanical-acoustic oscillation system. Mechanicalacoustic oscillations are generated in the piezo motor by a PZT-stack and transmitted to an oscillator element, and then from the oscillator element to a load action element. At high frequencies, when dimensions of the oscillator are proportionate to the wavelength, the energy is transmitted owing to acoustic waves. In this case, mechanical waves practically are not involved in the energy transmission process.

\footnotetext{
*Corresponding author: antonazin@niipmm.tsu.ru
} 


\section{Determine of the efficiency of the plane wave acoustic radiation in the multilayer structure}

Acoustic waves are a phenomenon characteristic for solid, liquid and gaseous media. The acoustic wave transmission (in general, oscillations) occurs systematically in full accordance with vibration of elastic bodies described by the oscillation equation. The acoustic wave transmission is described by the oscillation equation. The energy transmission efficiency is evaluated by the physical concept of the acoustic impedance [14].

Within the medium that does not contain external acoustic sources, the equation for a plane wave distributed in $\mathrm{X}$ direction will be:

$$
\left(\mathrm{d}^{2} /\left(\mathrm{d} x^{2}\right)+k^{2}\right) \Phi=0
$$

where: $k=\omega \cdot \sqrt{ }(\rho / x)=\omega / c=2 \pi / \lambda$ - wave number, $x$ - Young's modulus, $\lambda$ - wave length, $\omega$ - angular frequency of rotation, $c$ - acoustic velocity, $\Phi$ - efficiency of the source related to a unit volume by the velocity [5].

To determine the energy transmission efficiency of a travelling plane wave, it is necessary to calculate the acoustic impedance (2).

$$
Z=\mathrm{F} / \dot{\mathrm{X}}=\rho \cdot \mathrm{c}=\sqrt{ } \rho \cdot x,
$$

where: $\rho$ and $x$ - density and bulk elasticity coefficient (Young's modulus) of the layer, $\dot{X}-$ velocity potential in the layer, $F$ - force of influence of one medium on another. A plane wave is an ideal mechanism of the energy transmission. Let us consider the task of the plane waves, distribution in a layered medium formed by parallel to each other layers of the substance (Fig. 1).

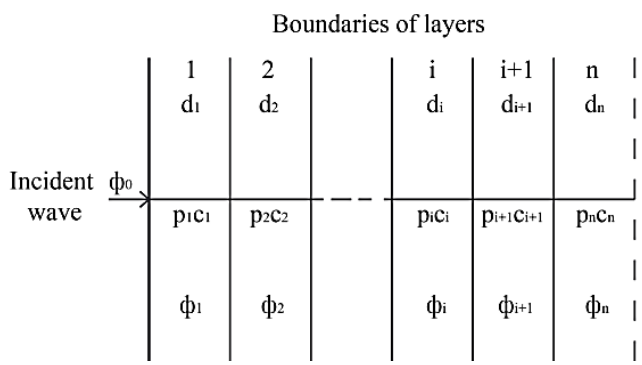

a)

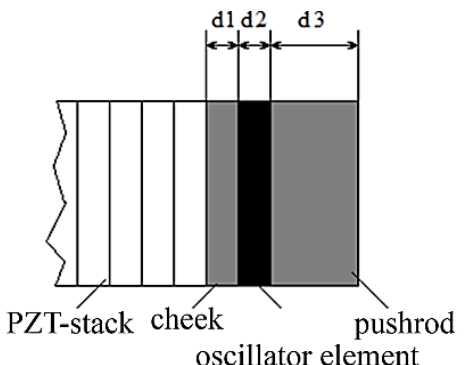

b)

Fig. 1. Distribution of a plane wave in the multilayer structure: a) distribution of a plane wave in the n-layer medium; b) oscillation system of the piezo motor in terms of the multilayer structure.

The task solution for a three-layer structure will define the efficiency of the acoustic power $\eta$ transmission. Let us calculate a ratio of the output signal W3 (the plane wave power in the third layer) to the input signal W0 (the plane wave power in the first layer) [5].

$$
\eta=\mathrm{W} 3 / \mathrm{W} 0=\left(4 \mathrm{R}_{1} \cdot \mathrm{R}_{2}^{2} \cdot \mathrm{R}_{3}\right) /\left(\left(\mathrm{R}_{1}+\mathrm{R}_{3}\right)^{2} \cdot \mathrm{R}_{2}{ }^{2} \cdot \cos ^{2} \theta_{2}+\left(\mathrm{R}_{2}{ }^{2}+\mathrm{R}_{1} \cdot \mathrm{R}_{2}\right)^{2} \cdot \sin ^{2} \theta_{2}\right) .
$$

Here $R_{i}=\rho_{i} \cdot c_{i}, \theta_{2}=k_{2} \cdot d_{2}, d_{2}$ - thickness of intermediate second layer.

The piezo motor design and materials are considered in terms of the multilayer structure under conditions of acoustic radiation (Fig. 1) [3, 4]. Materials with the low acoustic impedance are plexiglass and bakelite. Aluminium and titanium have the lowest acoustic impedance among metals. 
The material of cheeks, the oscillator element and the pushrod may vary, but at that time, the process of acoustic radiation of the plane wave will occur with a different efficiency.

The equation (3) is used for calculation of the efficiency of the plane wave acoustic radiation for materials with different wave impedances.

In the first case under consideration, aluminium is applied as a material for the cheek, oscillator element and the pushrod. The wave impedance of aluminium is $R_{\text {aluminium }}=$ $\rho_{i} \cdot c_{i}=1.377 \cdot 10^{7}\left[\mathrm{~kg} / \mathrm{sec} \cdot \mathrm{m}^{2}\right]$. The calculation shows that the piezo motor efficiency for identical wave impedances is equal to one, and the oscillator element length does not affect the radiation efficiency.

In the second case under consideration, aluminium is taken as a material for the cheek and the oscillator element, and the pushrod is made of plexiglass. The wave impedance of plexiglass is $R_{\text {plexiglass }}=\rho_{i} \cdot c_{i}=3.0 \cdot 10^{6}\left[\mathrm{~kg} / \mathrm{sec} \cdot \mathrm{m}^{2}\right]$. In this combination of materials, the system efficiency was reduced to $0.6(60 \%)$ in the low frequency area and became frequency dependent. With increase in the thickness of the oscillator element layer, the system efficiency gets low and reaches 0.2 (20\%) at frequencies above $100 \mathrm{kHz}$ (Fig. 2).

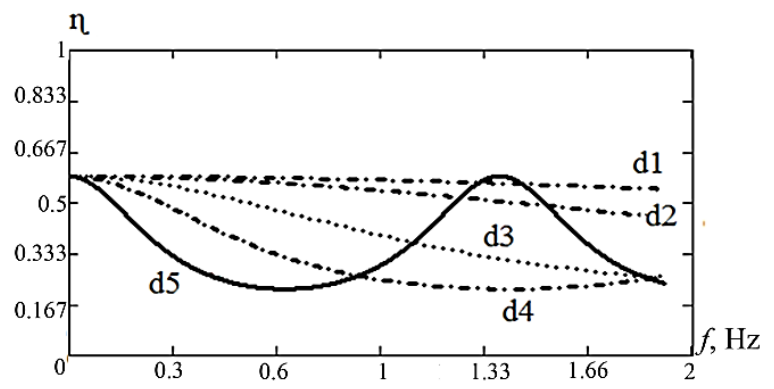

Fig. 2. Frequency response curve of the acoustic system efficiency. A material of the cheek and the oscillator element is aluminium; a material of the pushrod is plexiglass. The thickness of the oscillator element layer is: $d_{1}=0.92 \mathrm{~mm}, d_{2}=1.8 \mathrm{~mm}, d_{3}=4.6 \mathrm{~mm}, d_{4}=9 \mathrm{~mm}, d_{5}=18 \mathrm{~mm}$.

In the third case under consideration, plexiglass is taken as a material for the cheek and the oscillator element, and the pushrod is made of bakelite. The wave impedance of bakelite is $R_{\text {bakelite }}=\rho_{i} \cdot c_{i}=2.06 \cdot 10^{6}\left[\mathrm{~kg} / \mathrm{sec} \cdot \mathrm{m}^{2}\right]$. The wave impedance of plexiglass and bakelite are similar and the system efficiency with a small thickness of the oscillator element layer is about to one. Increasing the thickness of the oscillator element layer will reduce the system efficiency up to 0.7 , and for the thicknesses above $9 \mathrm{~mm}$ the efficiency is variable depending on the excitation frequency (Fig. 3).
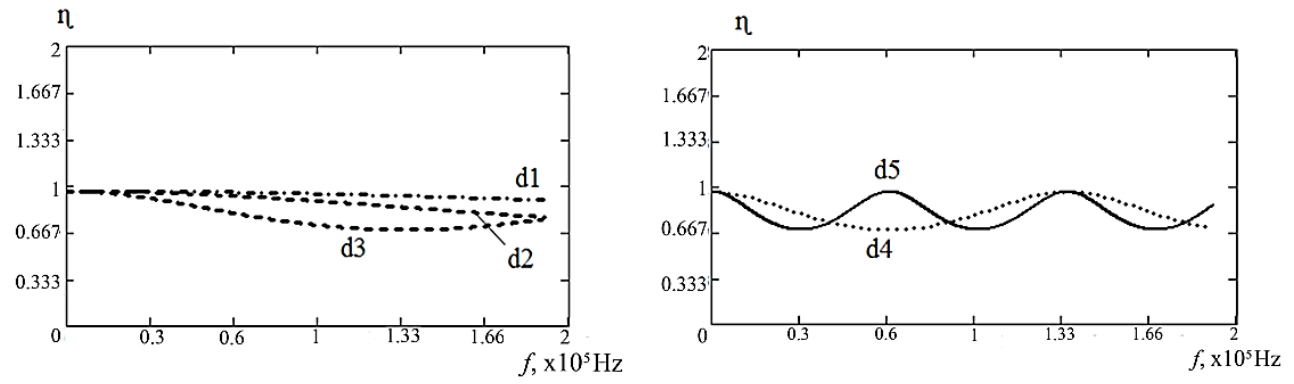

Fig. 3. Frequency response curves of the acoustic system efficiency. A material of the cheek and the oscillator element is plexiglass; a material of the pushrod is bakelite, and with the different thickness of the oscillator element layer is: $d_{1}=0.92 \mathrm{~mm}, d_{2}=1.8 \mathrm{~mm}, d_{3}=4.6 \mathrm{~mm}, d_{4}=9 \mathrm{~mm}, d_{5}=18 \mathrm{~mm}$. 
In the fourth case under consideration, Bakelite is taken as a material for the cheek; the oscillator element and the pushrod are made of plexiglass. According to the calculation done, it is determined that the system efficiency is 0.96 and does not change for different thicknesses of the oscillator element layer.

\section{Conclusion}

The developed calculation procedure allows for qualitative selection of materials for the pushrod elements design with the maximum efficiency of the acoustic energy transmission. To obtain the maximum efficiency, it is necessary that the wave impedances will be similar or close by their value; in this case, the efficiency is about to one. When the thickness of the oscillator element layer is increased, the system efficiency is reduced up to 0.7 ; and for the thickness above $9 \mathrm{~mm}$, the energy transmission efficiency is variable depending on the excitation frequency. The oscillator element thickness should be minimum and determined by the pushrod design peculiarities.

This work was financially supported by the Ministry of Education and Science of Russia; unique identifier RFMEFI57817X0257.

\section{References}

1. S. Park, S. He, Ultrasonics 52, 880 (2012)

2. Z. Wang, Aerospace Science and Technology, 160 (2013)

3. A.V. Azin, E.P. Bogdanov, S.V. Rikkonen, S.V. Ponomarev, A.M. Khramtsov, IOP Conf. Ser.: Mater. Sci. Eng 177012003 (2017)

4. A.V. Azin, S.V. Rikkonen, S.V. Ponomarev, A.M. Khramtsov, Proceedings of the Scientific-Practical Conference "Research and Development - 2016", 247 (2017)

5. T. Hayasaka Electroacoustics (Moscow: publishing house WORLD, 1982) 